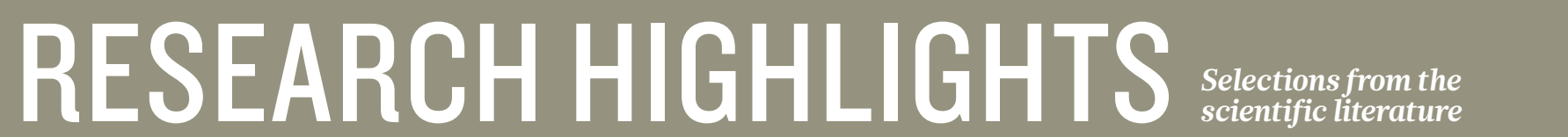

\section{MARINE METAGENOMICS}

\section{Sequencing from scratch}

Although most microorganisms cannot currently be cultured, their genomes may soon be accessible.

Until now, metagenomic analyses have been able to identify only dominant members of a microbial community or those sequenced previously. Virginia Armbrust and her group at the University of Washington in Seattle developed computational tools to tame the massive amount of data produced by next-generation sequencers. The method successfully sequenced two of 14 candidate genomes identified in samples from Puget Sound, most notably a microbe of low abundance but great interest - a representative of the mysterious, as yet uncultured organisms known as marine group II Euryarchaeota.

Researchers now have a way to peer into the secret lives of the uncultured majority.

Science 335, 587-590 (2012)

\section{MATERIALS}

\section{Printing tiny coiled antennas}

Typically, the largest circuit component in wireless electronic devices such as mobile phones is the antenna, which sends and receives electromagnetic waves. The tiniest antennas available are made up of wires twisted into three-dimensional coils to save

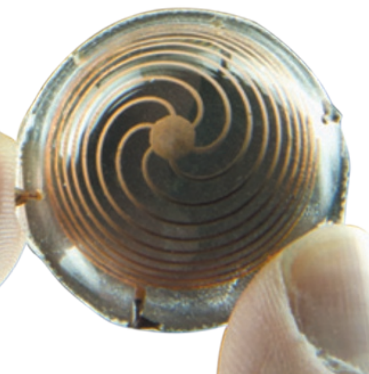

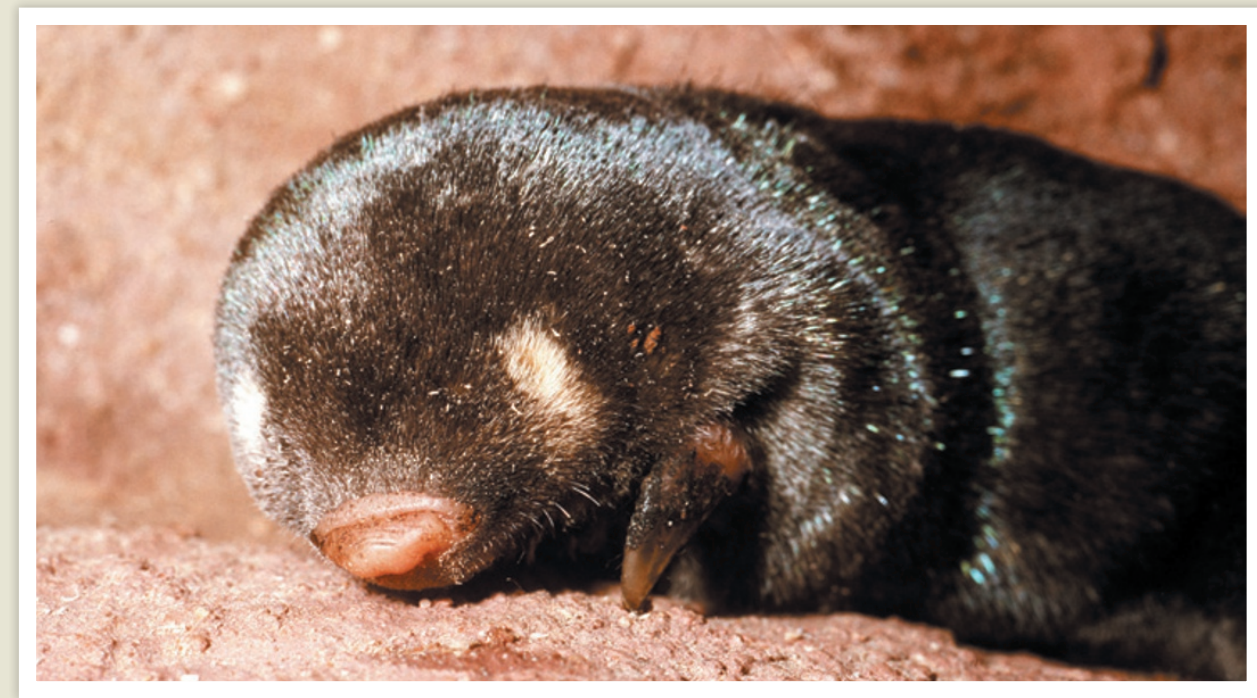

EVOLUTION

\section{Glad rags for a blind mole}

Golden moles have a blue-green sheen to their coats that is a rare example of iridescence in mammals, report Matthew Shawkey at the University of Akron in Ohio and his colleagues.

The group conducted the first detailed study of iridescent outer hairs and non-iridescent downy hairs from four species of golden mole. Iridescent hairs were highly flattened with much smaller scales than their less eye-catching counterparts. The scales form multiple layers, which alternate in colour between light and dark, and probably produce colour as light passes between layers in a phenomenon called thin-film interference.

All four mole species are blind, so it is unlikely that the hairs evolved as sexual ornamentation. The authors suggest that the iridescence of these burrowing animals is a by-product of adaptations for durable, low-friction pelts. Biol. Lett. http://dx.doi.org/10.1098/rsbl.2011.1168 (2012) on space while maintaining high radiation efficiency and wide bandwidth. But bending wires is cumbersome and expensive.

Stephen Forrest and Anthony Grbic at the University of Michigan in Ann Arbor and their colleagues report a way to rapidly transfer metallic patterns directly onto a curved polymer, which can be pre-moulded to a desired shape. Stamping the pattern onto a hemispherical polymer, for instance, produces miniature high-performance antennas curled in spherical helices (pictured).

Adv. Mater. http://dx.doi.org/10. 1002/adma/201104290 (2012)

\section{NETWORKS}

\section{Patchy}

\section{communication}

People tend to communicate with each other in bursts, exchanging clusters of messages over short time periods, and following these up with longer gaps in communication. But are these patterns simply the result of a tendency to talk more during the day and the working week?

Hang-Hyun Jo of Aalto University in Finland and his colleagues found that these temporal cycles are not sufficient to explain the bursts. They analysed 322 million mobile-phone calls between more than 5 million users over 119 days in 2007. After removing the effects of the day-night and working-week cycles, the bursts remained.

The authors suggest that the patterns reflect something fundamental in the way that people communicate. N. J. Phys. 14, 013055 (2012)

\section{GANCER DRUGS}

\section{Chemo spans generations}

Some commonly used cancer drugs not only generate mutations in treated mice, but scar the genomes of their 